\title{
AUDITORY IN-VEHICLE TECHNOLOGIES TO SUPPORT OLDER DRIVERS
}

\author{
Carryl L. Baldwin, Ian Reagan, \\ Jeffrey H. Lawrence, and T. Robert Turner \\ Department of Psychology \\ Old Dominion University \\ Norfolk, VA, USA \\ E-mail: cbaldwin@odu.edu
}

PAPER NOT AVAILABLE

\begin{abstract}
\section{OBJECTIVES}

Population aging, in combination with improved health care and more active lifestyles well into advanced age, have resulted in an increased number of older adults driving more miles than ever before. Unfortunately, these older drivers are over-represented in motor vehicle crashes and crash-related fatalities. Rather than the risk-tasking behaviors observed in young drivers, the collisions of older drivers frequently involve perceptual-cognitive errors. Advanced in-vehicle technologies have the potential to function as sensory-cognitive aids and may offset the negative impact of age-related changes in sensory and cognitive abilities. Collision Avoidance Systems (CASs) function as sensory aids to augment hazard detection capabilities, and therefore may be of particular benefit to older drivers. Navigation aids can offset the working memory requirements of wayfinding, and auditory guidance directions may reduce the visual demands of searching for street signs and reading maps. However, these advanced systems also have the potential to increase the information processing demands of the driving task or distract drivers, particularly if they are not designed in accordance with the sensory and perceptual capabilities of older adults. A series of experiments aimed at examining the impact of sensory-cognitive characteristics of auditory navigational aids on driver wayfinding, performance on a visual peripheral detection task, and neurophysiological, behavioral and subjective indices of driver mental workload and performance were conducted.
\end{abstract}

\section{METHODS}

Results of two investigations will be discussed. The first investigation examined the impact of amplitude level on working memory. Older adults frequently exhibit reduced complex working memory span. However, recent evidence indicates that increasing a sound's amplitude increases its duration in echoic memory (Baldwin, in press). Based on these findings, we hypothesized that increasing the amplitude of verbal material would improve working memory efficiency.

\section{RESULTS}

In support of this hypothesis, a strong positive correlation between the amplitude level at which the verbal material was presented and complex working memory span as measured by a version of Daneman and Carpenter's (1980) Listening span task was observed. This positive relationship 
was stronger in older relative to younger listeners. While young adults performance remained relatively unchanged across amplitude levels, older adults processed the verbal information less efficiently and had reduced retention of the presented material as amplitude level decreased even within a clearly audible range.

\section{CONCLUSIONS}

In a second investigation, amplitude of the verbal instructions affected not only verbal processing efficiency but also behavioral measures of driving performance (specifically lane variability). Results of both of these investigations are discussed in terms of their application and potential impact on the safety and effectiveness of auditory in-vehicle technologies to support older drivers.

Data is currently being collected on the impact of both the signal-to-noise ratio of voice guidance instructions against the background of simulated traffic noise and the presence or absence of a reference to a salient landmark in addition to standard turn-by-turn directions on young and older drivers' wayfinding behavior, navigational errors, driving performance and performance on a visual peripheral detection task. Explicitly pointing out salient landmarks has been shown to improve wayfinding performance in young drivers (Reagan \& Baldwin, 2006). It is hypothesized that salient visual landmarks will be of particular benefit to older drivers. Specifically, it is predicted that relative to the standard condition, when provided with a salient landmark both young and older drivers will make fewer navigational errors and will perform better (increased detection rate and reduced detection latency) on the peripheral detection task, as well as have fewer navigational errors and improved driving performance. A three-way interaction is expected between voice guidance format (standard and landmark), amplitude level, and age, such that the presence of the landmark is expected to be of particular benefit to older relative to young drivers in the low-amplitude condition. However, insufficient data from older participants has been collected to date to allow for statistical analyses of these predictions.

\section{REFERENCES}

Baldwin, C.L. (In press). Cognitive implications of echoic persistence. Memory \& Cognition.

Reagan, I., and Baldwin, C.L. (2006). Facilitating route memory with auditory route guidance systems. Journal of Environmental Psychology, 26(2), 146-155. 